\title{
Weighted exponential stability for generalized delay functional differential equations with bounded delays
}

\author{
Snezhana Hristova ${ }^{1 *}$ and Valentina Proytcheva ${ }^{2}$
}

*Correspondence:

snehri@uni-plovdiv.bg

1 Plovdiv University, Plovdiv, Bulgaria

Full list of author information is

available at the end of the article

\begin{abstract}
In the paper functional differential equations with several bounded delays are considered. The delays are presented in the form of delay operators and they generalize many well-known types of delay differential equations in the literature, such as differential equations with constant delays, with variable delays, with distributed delays, differential equations with maxima, etc. A special type of stability, known as $\psi$-exponential stability, is studied and several sufficient conditions are obtained. The used function $\psi$ plays the role of a weight in the norm and, additionally, it gives the rate of increase of the solutions which are not exponentially stable in the regular sense. The modified Razumikhin method as well as comparison results have been applied. Several examples illustrate the results obtained.
\end{abstract}

MSC: $34 \mathrm{~K} 20$

Keywords: exponential stability with weight; Lyapunov function; delay; differential equations

\section{Introduction}

One of the main qualitative questions in the theory of differential equations is stability. The problems of the stability of the solutions of differential equations via Lyapunov functions have been successfully investigated and various types of stability have been introduced. One of the useful types of stability is the so-called exponential stability. In $[1,2]$ the authors have investigated the exponential stability of impulsive delay differential equations by using the method of Lyapunov functions and by Razumikhin techniques.

The exponential stability gives information as regards the rate of decay of the solutions only in the case when the solutions are stable. But often the solutions are not bounded at all, or they are bounded but not exponentially stable. In this case we could apply a weight such that the solution could become exponentially stable in a different sense. We could introduce and use the so-called $\psi$-stability. The notion of $\psi$-stability of degree $k$ for ordinary differential equations has been introduced by Akinyele [3]. Also various types of $\psi$-stability have been studied for nonlinear systems of ordinary differential equations [4], for nonlinear Volterra integro-differential systems [5], for impulsive differential equations [6]. Meanwhile, in [6] points in $\mathbb{R}^{n}$ are incorrectly mixed with values of functions with a range $\mathbb{R}^{n}$ and the weights in the examples are useless.

○2014 Hristova and Proytcheva; licensee Springer. This is an Open Access article distributed under the terms of the Creative Commons Attribution License (http://creativecommons.org/licenses/by/2.0), which permits unrestricted use, distribution, and reproduction in any medium, provided the original work is properly cited. 
In the mathematical simulation in various important branches of control theory, pharmacokinetics, economics, etc., one has to analyze the influence of the deviation of the regulated quantity. Such a kind of problems could be adequately modeled by differential equations that contain a delay operator. In this paper differential equations with a special type of delay operators are considered. These delay equations generalize many types of equations well known in the literature. Several sufficient conditions for the $\psi$-exponential stability by scalar Lyapunov functions are obtained. The modified Razumikhin method and comparison results have been applied. Several examples, solved and graphed by Wolfram Mathematica, are given to illustrate the main concepts of the weighted exponential stability.

\section{Preliminary notes and definitions}

Let $r>0$ be a given number. Define the operators $G_{k}: C\left([-r, \infty), \mathbb{R}^{n}\right) \rightarrow \mathbb{R}^{n}, k=1,2, \ldots, m$, such that for any function $x \in C\left([-r, \infty), \mathbb{R}^{n}\right)$, any point $t \in \mathbb{R}_{+}$, and $k=1,2, \ldots, m$, there exists a point $\xi \in[t-r, t]$, depending on $x, t, k$, such that $G_{k}(x)(t)=C x(\xi)$, where $C$ is a constant.

Consider the nonlinear generalized delay functional differential equations with bounded delays

$$
x^{\prime}=f\left(t, x(t), G_{1}(x)(t), G_{2}(x)(t), \ldots, G_{m}(x)(t)\right) \quad \text { for } t \geq t_{0},
$$

with initial condition

$$
x\left(t+t_{0}\right)=\varphi(t) \quad \text { for } t \in[-r, 0],
$$

where $x \in \mathbb{R}^{n}, f: \mathbb{R}_{+} \times \mathbb{R}^{n} \times \mathbb{R}^{n m} \rightarrow \mathbb{R}^{n}, t_{0} \in \mathbb{R}_{+}, \varphi:[-r, 0] \rightarrow \mathbb{R}^{n}$.

We would like to emphasize some particular cases of (1):

- if for any point $t \in \mathbb{R}_{+}$the point $\xi_{k} \in \mathbb{R}$ is such that $\xi_{k}=t-h_{k}, k=1,2, \ldots, m$, where $h_{k}=$ const $\in[0, r], k=1,2, \ldots, m$, then $G_{k}(x)(t)=x\left(t-h_{k}\right)$ for $t \in \mathbb{R}_{+}$and (1) is reduced to the delay differential equations with constant delays, well known and studied in the literature, $x^{\prime}=f\left(t, x(t), x\left(t-h_{1}\right), x\left(t-h_{2}\right), \ldots, x\left(t-h_{m}\right)\right)$;

- if for any point $t \in \mathbb{R}_{+}$the point $\xi_{k} \in \mathbb{R}$ is such that $\max _{s \in\left[t-h_{k}, t\right]} x(s)=x\left(\xi_{k}\right)$, $h_{k}=$ const $\in[0, r], k=1,2, \ldots, m$, then $G_{k}(x)(t)=\max _{s \in\left[t-h_{k}, t\right]} x(s)$ for $t \in \mathbb{R}_{+}$and (1) is reduced to differential equations with maxima, which are partially studied in [7-13], see also the monograph [14] and the references cited therein:

$$
x^{\prime}=f\left(t, x(t), \max _{s \in\left[t-h_{1}, t\right]} x(s), \max _{s \in\left[t-h_{2}, t\right]} x(s), \ldots, \max _{s \in\left[t-h_{m}, t\right]} x(s)\right)
$$

- if for any point $t \in \mathbb{R}_{+}$the point $\xi_{k} \in \mathbb{R}$ is such that $\xi_{k}=t-\tau_{k}(t)$, where the functions $\tau_{k}: \mathbb{R}_{+} \rightarrow[0, r]$, then $G_{k}(x)(t)=x\left(t-\tau_{k}(t)\right)$ for $t \in \mathbb{R}_{+}$and (1) is reduced to the delay differential equations with variable bounded delays, well known and studied in the literature: $x^{\prime}=f\left(t, x(t), x\left(t-\tau_{1}(t)\right), x\left(t-\tau_{2}(t)\right), \ldots, x\left(t-\tau_{m}(t)\right)\right)$ (for example, $\tau(t)=r|\sin (t)|$ or $\tau(t)=\frac{r t}{t+1}$ for $\left.t \in \mathbb{R}_{+}\right)$.

In our work we will assume that IVP (1), (2) (initial value problem) has a solution $x\left(t ; t_{0}, \varphi\right)$ defined on $\left[t_{0}-r, \infty\right)$ for any $t_{0} \in \mathbb{R}_{+}$and $\varphi \in C\left([-r, 0], \mathbb{R}^{n}\right)$. 
In our investigations we will use a special function, which will play the role of the weight in the regular norm in $\mathbb{R}^{n}$ and it will help us to generalize the well-known exponential stability. Let $\psi_{k}:[-r, \infty) \rightarrow(0, \infty), k=1,2, \ldots, n$, be given continuous functions and let $\psi$ be a $n \times n$-dimensional matrix defined by $\psi=\operatorname{diag}\left[\psi_{1}, \psi_{2}, \ldots, \psi_{n}\right]$.

We will define exponential stability with a weight for generalized nonlinear delay functional differential equations (1).

Definition 1 Let the functions $\psi_{k} \in C([-r, \infty),(0, \infty)), k=1,2, \ldots, n$, be given. The zero solution of the system of generalized delay functional differential equations (1) is said to be:

(1) $\psi$-exponentially stable, if for any initial point $t_{0} \in \mathbb{R}_{+}$and any initial function $\varphi \in C\left([-r, 0], \mathbb{R}^{n}\right)$ there exist $\beta=\beta\left(t_{0}\right)>0$ and a constant $\delta>0$ such that the solution $x\left(t ; t_{0}, \varphi\right)$ of IVP (1), (2) satisfies

$$
\left\|\psi(t) x\left(t ; t_{0}, \varphi\right)\right\| \leq \beta\left(t_{0}\right)\|\psi \varphi\|_{t_{0}} e^{-\delta\left(t-t_{0}\right)}, \quad t \geq t_{0},
$$

where $\|\psi \varphi\|_{t_{0}}=\max _{s \in\left[t_{0}-r, t_{0}\right]}\left\|\psi(s) \varphi\left(s-t_{0}\right)\right\|$;

(2) $\psi$-uniformly exponentially stable, if $\beta$ in (1) does not depend on $t_{0}$.

Remark 1 The definition of stability given above generalizes the exponential stability, well known in the literature (if $\psi_{k}(t) \equiv 1$ for $k=1,2, \ldots, n$ ).

Remark 2 If the weight function $\psi$ is one-to-one, then we could consider the substitution $y(t)=\psi(t) x(t)$, where $x(t)$ is a solution of (1), and study the exponential stability of $y(t)$ which is equivalent to the $\psi$-exponential stability of $x(t)$. But if the function $\psi(t)$ is not a one-to-one function, then we have to study directly the behavior of the solution (1).

The $\psi$-exponential stability defined above is often used in connection with the rate of increase/decrease of unbounded solutions.

Definition 2 The rate of increase of a function $u:[a, \infty) \rightarrow \mathbb{R}^{n}$ is smaller than the function $\eta \in C([a, \infty),(0, \infty))$ if there exists $C=C(a, u(a))>0$ such that $\|u(t)\|<C \eta(t)$ for $t \geq a$.

Remark 3 If the zero solution of (1) is exponentially stable, i.e.

$$
\left\|x\left(t ; t_{0}, \varphi\right)\right\|<\beta\left(t_{0}\right)\|\varphi\|_{t_{0}} e^{-\delta\left(t-t_{0}\right)}, \quad t \geq t_{0}
$$

then any solution of (1) has a rate of increase smaller than $e^{-\delta t}$, where $C=\beta\left(t_{0}\right)\|\varphi\|_{t_{0}} e^{\delta t_{0}}$.

Remark 4 If the solutions of (1) are unbounded but the zero solution is weighted exponentially stable, then with the help of the weight we could easily obtain the rate of increase of the solutions.

Remark 5 If the weight function is $\psi$ and we consider the substitution $y(t)=\psi(t) x(t)$, where $x(t)$ is a solution of (1), then the function $y(t)$ could satisfy a more complicated equation than the given one. Therefore, the study of the exponential stability of the zero 
solution of the equation could be more difficult than the study of the $\psi$-exponential stability of the given one. That is why it requires directly studying of the $\psi$-exponential stability and obtaining of appropriate sufficient conditions.

Example 1 Consider the delay differential equation

$$
x^{\prime}(t)=x(t-1) \quad \text { for } t \geq 2
$$

with an initial condition

$$
x(t+2)=\varphi(t) \quad \text { for } t \in[-1,0]
$$

where $x \in \mathbb{R}, \varphi \in C([-1,0], \mathbb{R})$.

Let $\varphi(t)=t^{2}$ for $t \in[-1,0]$. Then the solution $x(t)$ is unbounded.

Consider the function $\psi(t)=2^{-1.22 t}$. Then the function $y(t)=2^{-1.22 t} x(t)$ satisfies $y^{\prime}(t)=$ $-1.22 y(t)+2^{-1.22} y(t-1)$, which is more complicated than the given equation (3). That is why it is better to study directly the $\psi$-exponential stability of the zero solution of (3) (see Figure 1). Also, we could find the rate of increase of $x(t)$ which is smaller than the function $2^{1.22 t} e^{-0.22 t}$ (see Figure 2).

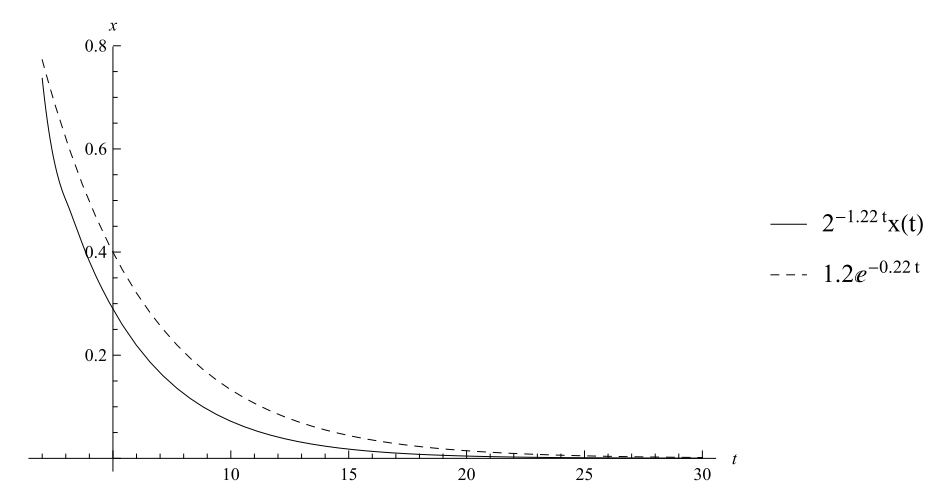

Figure 1 Graph of $\left|2^{-1.22 t} x(t)\right|$ and $1.2 e^{-0.22 t}$ on $[2,30]$.

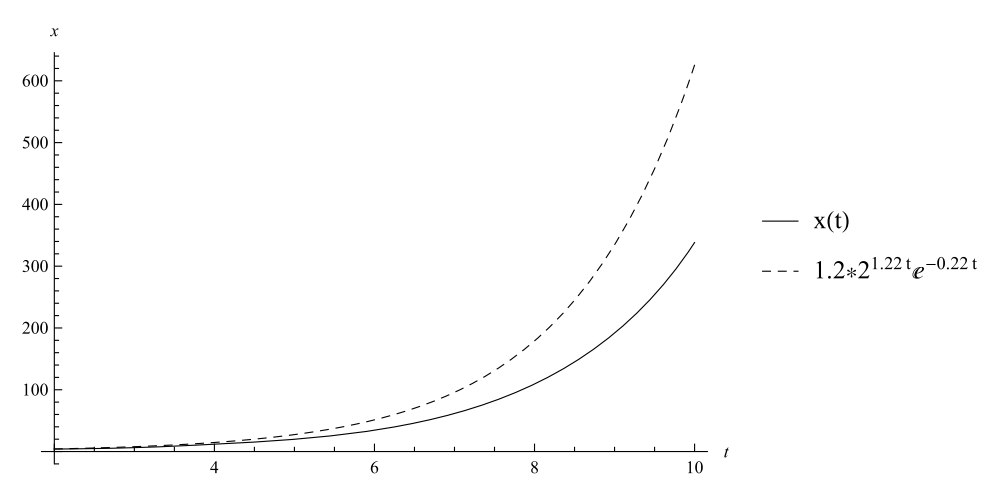

Figure 2 Graph of $|x(t)|$ and (1.2)2 $2^{1.22 t} e^{-0.22 t}$ on $[2,10]$. 


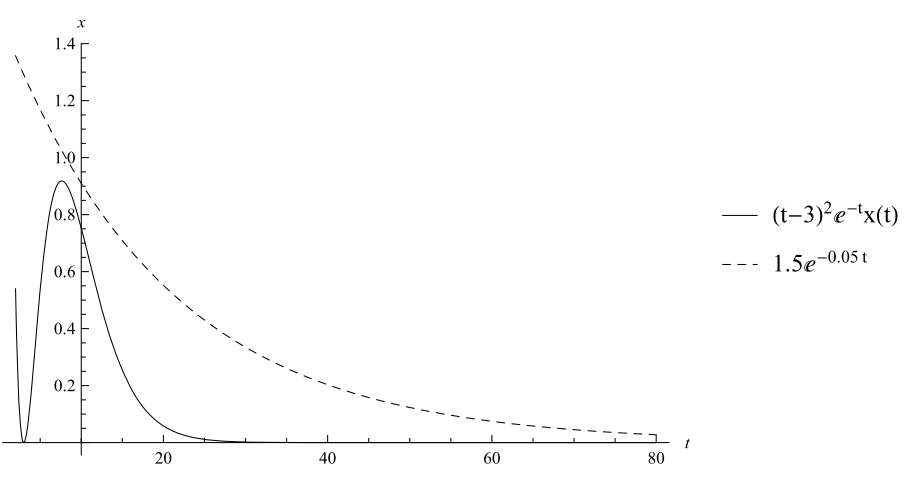

Figure 3 Graph of $\left|(t-3)^{2} e^{-t} x(t)\right|$ and $1.5 e^{-0.05 t}$ on $[2,80]$.

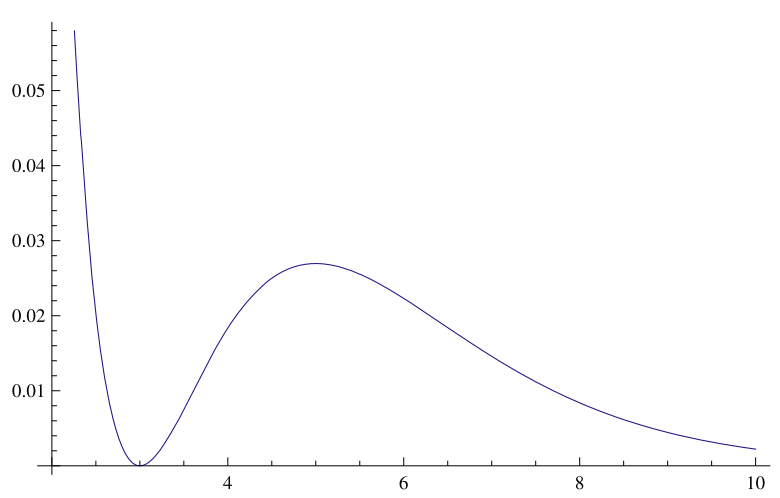

Figure 4 Graph of $(t-3)^{2} e^{-t}$ on $[2,10]$.

Now let the function $\psi(t)=(t-3)^{2} e^{-t}$. Obviously the function $y(t)=\psi(t) x(t)$ satisfies a more complicated equation. We directly graph the functions $\psi(t) x(t)$ and $1.5 e^{-0.05 t}$ where $x(t)$ is the solution of (3) (see Figure 3). The zero solution could be $\left\{(t-3)^{2} e^{-t}\right\}$ exponentially stable. In this case, since the function $\psi(t)$ is not a one-to-one function (Figure 4) we are not able to find directly the rate of increase of $x(t)$.

We will use Lyapunov functions and the Razumikhin method to obtain some sufficient conditions for the $\psi$-exponential stability of the zero solution of (1). We will apply the differentiable Lyapunov function $V(t, x)$ and we will define for any $t \in \mathbb{R}_{+}$and any function $\Psi \in C\left([-r, 0], \mathbb{R}^{n}\right)$ a derivative of $V(t, x)$ along a trajectory of the system (1) with a weight $\psi$ as follows:

$$
\begin{aligned}
D_{(1)} V(t, \psi(t) \Psi(0)) \\
=\frac{\partial V(t, \psi(t) \Psi(0))}{\partial t} \\
\quad+\frac{\partial V(t, \psi(t) \Psi(0))}{\partial x}\left(\psi^{\prime}(t) \Psi(0)\right. \\
\left.\quad+\psi(t) f\left(t, \Psi(0), G_{1}(\Psi)(0), G_{2}(\Psi)(0), \ldots, G_{m}(\Psi)(0)\right)\right),
\end{aligned}
$$

where $\psi^{\prime}(t)=\operatorname{diag}\left[\psi_{1}^{\prime}(t), \psi_{2}^{\prime}(t), \ldots, \psi_{n}^{\prime}(t)\right]$. 


\section{Main results}

We will obtain some sufficient conditions for the weighted exponential stability of nonlinear generalized delay functional differential equations.

Theorem 1 Let there exist a Lyapunov function $V \in C^{1}\left([-r, \infty) \times \mathbb{R}^{n}, \mathbb{R}_{+}\right)$, functions $a, b$ : $[-r, \infty) \rightarrow \mathbb{R}_{+}, w: \mathbb{R}_{+} \rightarrow(0, \infty)$, and a positive constant $p$ such that $\inf _{t \geq 0} w(t)=C>0$, $\inf _{t \geq 0} a(t)=A>0$, and

(i) $a(t)\|\psi(t) x\|^{p} \leq V(t, x) \leq b(t)\|\psi(t) x\|^{p}$ for $t \in[-r, \infty), x \in \mathbb{R}^{n}$;

(ii) for any number $t \in \mathbb{R}_{+}$and any function $\phi \in C\left([-r, 0], \mathbb{R}^{n}\right)$ such that $V(t, \phi(0)) \geq e^{-\int_{t-r}^{t} w(s) d s} V(t+s, \phi(s))$ for $s \in[-r, 0)$ the inequality

$$
D_{(1)} V(t, \phi(0))<-w(t) V(t, \phi(0))
$$

holds.

Then the zero solution of the generalized delay functional differential equation (1) is $\psi$-exponentially stable.

Proof Consider a solution $x(t)=x\left(t ; t_{0}, \varphi\right)$ of IVP (1), (2) for $\varphi \in C\left([-r, 0], \mathbb{R}^{n}\right), t_{0} \in \mathbb{R}_{+}$.

Define functions $M:\left[t_{0}-r, \infty\right) \rightarrow \mathbb{R}_{+}$and $Q:\left[t_{0}-r, \infty\right) \rightarrow \mathbb{R}$ by the equalities

$$
M(t)= \begin{cases}\max _{s \in[t-r, t]} b(s) & \text { for } t \geq t_{0}, \\ \max _{s \in\left[t_{0}-r, t\right]} b(s) & \text { for } t \in\left[t_{0}-r, t_{0}\right]\end{cases}
$$

and

$$
Q(t)= \begin{cases}V(t, x(t))-M\left(t_{0}\right)\left(\|\psi \varphi\|_{t_{0}}\right)^{p} e^{-\int_{t_{0}}^{t} w(s) d s} & \text { for } t \geq t_{0}, \\ V\left(t, \varphi\left(t-t_{0}\right)\right)-M\left(t_{0}\right)\left(\|\psi \varphi\|_{t_{0}}\right)^{p} & \text { for } t \in\left[t_{0}-r, t_{0}\right] .\end{cases}
$$

We will prove the function $Q(t)$ to be non-positive.

Let $t \in\left[t_{0}-r, t_{0}\right]$. Then from condition (i) we get $Q(t)=V\left(t, \varphi\left(t-t_{0}\right)\right)-M\left(t_{0}\right)\left(\|\psi \varphi\|_{t_{0}}\right)^{p} \leq$ $b(t)\left\|\psi(t) \varphi\left(t-t_{0}\right)\right\|^{p}-M\left(t_{0}\right)\left(\|\psi \varphi\|_{t_{0}}\right)^{p} \leq 0$. Therefore, the function $Q(t) \leq 0$ on $\left[t_{0}-r, t_{0}\right]$.

Assume the inequality $Q(t) \leq 0$ does not hold for $t>t_{0}$ and let $t^{*}=\sup \left\{t \geq t_{0}: Q(s) \leq\right.$ 0 for $\left.s \in\left[t_{0}, t\right]\right\}$. It is clear that $t^{*}<\infty, Q\left(t^{*}\right)=0$, and

$$
Q^{\prime}\left(t^{*}\right) \geq 0
$$

Case 1. Let $t^{*}-r<t_{0}$.

For $s \in\left[-r, t_{0}-t^{*}\right)$ we have

$$
\begin{aligned}
& V\left(t^{*}+s, x\left(t^{*}+s\right)\right) e^{-\int_{t^{*}-r}^{t^{*}} w(s) d s} \\
& \quad=\left(Q\left(t^{*}+s\right)+M\left(t_{0}\right)\left(\|\psi \varphi\|_{t_{0}}\right)^{p}\right) e^{-\int_{t^{*}-r}^{t^{*}} w(s) d s} \\
& \quad \leq\left(Q\left(t^{*}\right)+M\left(t_{0}\right)\left(\|\psi \varphi\|_{t_{0}}\right)^{p} e^{-\int_{t_{0}}^{t^{*}} w(s) d s}\right) e^{-\int_{t^{*}-r}^{t_{0}} w(s) d s} \\
& \quad<V\left(t^{*}, x\left(t^{*}\right)\right) .
\end{aligned}
$$


For $s \in\left[t_{0}-t^{*}, 0\right)$ and $t^{*}>t_{0}$ we get

$$
\begin{aligned}
& V\left(t^{*}+s, x\left(t^{*}+s\right)\right) e^{-\int_{t^{*}-r}^{t^{*}} w(s) d s} \\
& \quad=\left(Q\left(t^{*}+s\right)+M\left(t_{0}\right)\left(\|\psi \varphi\|_{t_{0}}\right)^{p} e^{-\int_{t_{0}}^{t^{*}+s} w(s) d s}\right) e^{-\int_{t^{*}-r}^{t^{*}} w(s) d s} \\
& \quad \leq\left(Q\left(t^{*}\right)+M\left(t_{0}\right)\left(\|\psi \varphi\|_{t_{0}}\right)^{p} e^{-\int_{t_{0}}^{t^{*}} w(s) d s}\right) e^{-\int_{t^{*}-r}^{t^{*}+s} w(s) d s} \\
& \quad<V\left(t^{*}, x\left(t^{*}\right)\right) .
\end{aligned}
$$

Case 2. Let $t^{*}-r \geq t_{0}$.

For $s \in[-r, 0)$ we have

$$
\begin{aligned}
& V\left(t^{*}+s, x\left(t^{*}+s\right)\right) e^{-\int_{t^{*}-r}^{t^{*}} w(s) d s} \\
& \quad=\left(Q\left(t^{*}+s\right)+M\left(t_{0}\right)\left(\|\psi \varphi\|_{t_{0}}\right)^{q} e^{-\int_{t_{0}}^{t^{*}+s} w(s) d s}\right) e^{-\int_{t^{*}-r}^{t^{*}} w(s) d s} \\
& \quad \leq M\left(t_{0}\right)\left(\|\psi \varphi\|_{t_{0}}\right)^{q} e^{-\int_{t_{0}}^{t^{*}} w(s) d s} e^{-\int_{t^{*}-r}^{t^{*}+s} w(s) d s} \\
& \quad \leq Q\left(t^{*}\right)+M\left(t_{0}\right)\left(\|\psi \varphi\|_{t_{0}}\right)^{p} e^{-\int_{t_{0}}^{t^{*}} w(s) d s} \\
& \quad=V\left(t^{*}, x\left(t^{*}\right)\right)
\end{aligned}
$$

According to the proved inequalities in Case 1 and Case 2 it follows that the inequality in condition (ii) is satisfied for the function $\phi(s)=x\left(t^{*}+s\right), s \in[-r, 0]$. Therefore, from inequality (5) we get

$$
\begin{aligned}
Q^{\prime}\left(t^{*}\right) & =D_{(1)} V\left(t^{*}, x\left(t^{*}\right)\right)+w\left(t^{*}\right) M\left(t_{0}\right)\left(\|\psi \varphi\|_{t_{0}}\right)^{p} e^{-\int_{t_{0}}^{t^{*}} w(s) d s} \\
& <-w\left(t^{*}\right)\left(V\left(t^{*}, x\left(t^{*}\right)\right)-M\left(t_{0}\right)\left(\|\psi \varphi\|_{t_{0}}\right)^{p} e^{-\int_{t_{0}}^{t^{*}} w(s) d s}\right) \\
& =-w\left(t^{*}\right) Q\left(t^{*}\right)=0 .
\end{aligned}
$$

Inequality (9) contradicts (8).

Therefore, for $t \geq t_{0}$ we have $V(t, x(t)) \leq M\left(t_{0}\right)\left(\|\psi \varphi\|_{t_{0}}\right)^{p} e^{-\int_{t_{0}}^{t} w(s) d s} \leq M\left(t_{0}\right)\left(\|\psi \varphi\|_{t_{0}}\right)^{p} \times$ $e^{-C\left(t-t_{0}\right)}$ and

$$
\begin{aligned}
\|\psi(x) x(t)\| & \leq \sqrt[p]{\frac{V(t, x(t))}{a(t)}} \leq \sqrt[p]{\frac{M\left(t_{0}\right)}{a(t)}}\left(\|\psi \varphi\|_{t_{0}}\right) e^{-\frac{C}{p}\left(t-t_{0}\right)} \\
& \leq \sqrt[p]{\frac{M\left(t_{0}\right)}{A}}\left(\|\psi \varphi\|_{t_{0}}\right) e^{-\frac{C}{p}\left(t-t_{0}\right)} \quad \text { for } t \geq t_{0}
\end{aligned}
$$

The proof of Theorem 1 is completed.

Remark 6 Note that the function $w(t)$ in inequality (5) could be replaced by a positive constant $C$.

Corollary 1 Let there exist positive constants $A, B, C, p$ such that $a(t) \equiv A, b(t) \equiv B, w(t) \equiv$ $C$ and the conditions of Theorem 1 are satisfied. 
Then the zero solution of the generalized delay functional differential equation (1) is $\psi$-uniformly exponentially stable.

In the case when the weight is involved in the derivative of the Lyapunov function we obtain the following sufficient conditions for the $\psi$-exponential stability.

Theorem 2 Let there exist a Lyapunov function $V \in C^{1}\left([-r, \infty) \times \mathbb{R}^{n}, \mathbb{R}_{+}\right)$, functions $a, b$ : $[-r, \infty) \rightarrow \mathbb{R}_{+}, w: R_{+} \rightarrow(0, \infty)$, and a positive constant $p$ such that $\inf _{t \geq 0} w(t)=C>0$, $\inf _{t \geq 0} a(t)=A>0$, and

(i) $a(t)\|x\|^{p} \leq V(t, x) \leq b(t)\|x\|^{p}$ for $t \in[-r, \infty), x \in \mathbb{R}^{n}$;

(ii) for any number $t \in \mathbb{R}_{+}$and any function $\phi \in C\left([-r, 0], \mathbb{R}^{n}\right)$ such that $V(t, \psi(t) \phi(0)) \geq e^{-\int_{t-r}^{t} w(s) d s} V(t+s, \psi(t+s) \phi(s))$ for $s \in[-r, 0)$ the inequality

$$
D_{(1)} V(t, \psi(t) \phi(0))<-w(t) V(t, \psi(t) \phi(0))
$$

holds.

Then the zero solution of the generalized delay functional differential equation (1) is $\psi$-exponentially stable.

Proof The proof of Theorem 2 is similar to the one of Theorem 1, but the function $Q(t)$ is defined by

$$
Q(t)= \begin{cases}V(t, \psi(t) x(t))-M\left(t_{0}\right)\left(\|\psi \varphi\|_{t_{0}}\right)^{p} e^{-\int_{t_{0}}^{t} w(s) d s} & \text { for } t \geq t_{0}, \\ V\left(t, \psi(t) \varphi\left(t-t_{0}\right)\right)-M\left(t_{0}\right)\left(\|\psi \varphi\|_{t_{0}}\right)^{p} & \text { for } t \in\left[t_{0}-r, t_{0}\right] .\end{cases}
$$

Remark 7 The function $w(t)$ under the conditions of Theorem 1 and Theorem 2 could not vanish, so in the case when the derivative of the Lyapunov function is strongly negative, we need another type of sufficient condition.

Theorem 3 Let there exist a Lyapunov function $V \in C^{1}\left([-r, \infty) \times \mathbb{R}^{n}, \mathbb{R}_{+}\right)$, functions $a, b$ : $[-r, \infty) \rightarrow \mathbb{R}_{+}: \inf _{t \geq 0} a(t)=A>0$, and positive constants $\gamma \in(0,1)$ and $p>0$ such that

(i) $a(t)\|x\|^{p} \leq V(t, x) \leq b(t)\|x\|^{p}$ for $x \in \mathbb{R}^{n}$;

(ii) for any number $t \in \mathbb{R}_{+}$and any function $\phi \in C\left([-r, 0], \mathbb{R}^{n}\right)$ such that $V(t, \psi(t) \phi(0))>\gamma V(t+s, \psi(t+s) \phi(s))$ for $s \in[-r, 0)$ the inequality

$$
D_{(1)} V(t, \psi(t) \phi(0))<0
$$

holds.

Then the zero solution of the generalized delay functional differential equation (1) is $\psi$-exponentially stable.

Proof Let $x(t)=x\left(t ; t_{0}, \varphi\right)$ be a solution of IVP (1), (2).

Choose a positive number $\lambda: \lambda<-\frac{\ln \gamma}{r}$. Then $\gamma e^{\lambda r}<1$.

Define a function $v:\left[t_{0}-r, \infty\right) \rightarrow \mathbb{R}_{+}$by $v(t)=V(t, \psi(t) x(t))$. We will prove

$$
v(t) \leq M\left(t_{0}\right)\|\psi \varphi\|_{t_{0}}^{p} e^{-\lambda\left(t-t_{0}\right)}, \quad t \geq t_{0}-r
$$

where the function $M(t)$ is defined by (6). 
Let $t \in\left[t_{0}-r, t_{0}\right]$. From condition (i) it follows that $v(t)=V(t, \psi(t) x(t)) \leq b(t)(\| \psi(t) \varphi(t-$ $\left.\left.t_{0}\right) \|\right)^{p} \leq M\left(t_{0}\right)\|\psi \varphi\|_{t_{0}}^{p} \leq e^{-\lambda\left(t-t_{0}\right)} M\left(t_{0}\right)\|\psi \varphi\|_{t_{0}}^{p}$.

Assume the contrary and let

$$
t^{*}=\sup \left\{t>t_{0}: v(s) \leq M\left(t_{0}\right)\|\psi \varphi\|_{t_{0}}^{p} e^{-\lambda\left(t-t_{0}\right)} \text { for } s \in\left[t_{0}, t\right]\right\} .
$$

It is obvious that $t^{*}<\infty, v\left(t^{*}\right) \leq M\left(t_{0}\right)\|\psi \varphi\|_{t_{0}}^{p} e^{-\lambda\left(t^{*}-t_{0}\right)}$ and

$$
v^{\prime}\left(t^{*}\right) \geq 0 \text {. }
$$

Let $s \in[-r, 0)$. Then we have

$$
\begin{aligned}
& \gamma V\left(t^{*}+s, \psi\left(t^{*}+s\right) x\left(t^{*}+s\right)\right) \\
& \quad=\gamma v\left(t^{*}+s\right) \leq \gamma M\left(t_{0}\right)\|\psi \varphi\|_{t_{0}}^{p} e^{-\lambda\left(t^{*}+s-t_{0}\right)} \\
& \quad \leq \gamma e^{\gamma r} M\left(t_{0}\right)\|\psi \varphi\|_{t_{0}}^{p} e^{-\lambda\left(t^{*}-t_{0}\right)} \\
& \quad=\gamma e^{\lambda r} v\left(t^{*}\right)<V\left(t^{*}, \psi\left(t^{*}\right) x\left(t^{*}\right)\right) .
\end{aligned}
$$

Therefore, from (ii) we get $D_{(1)} V\left(t^{*}, \psi\left(t^{*}\right) x\left(t^{*}\right)\right)<0$, which contradicts (12).

From inequality (11) and condition (i) it follows that

$$
\begin{aligned}
\|\psi(x) x(t)\| & \leq \sqrt[p]{\frac{V(t, \psi(t) x(t))}{a(t)}} \leq \sqrt[p]{\frac{M\left(t_{0}\right)}{a(t)}}\left(\|\psi \varphi\|_{t_{0}}\right) e^{-\frac{\lambda}{p}\left(t-t_{0}\right)} \\
& \leq \sqrt[p]{\frac{M\left(t_{0}\right)}{A}}\left(\|\psi \varphi\|_{t_{0}}\right) e^{-\frac{\lambda}{p}\left(t-t_{0}\right)} \quad \text { for } t \geq t_{0} .
\end{aligned}
$$

The proof of Theorem 3 is completed.

Corollary 2 Let conditions of Theorem $2 /$ Theorem 3 be satisfied for $a(t) \equiv A>0$ and $b(t) \equiv b>0$.

Then the zero solution of the generalized delay functional differential equation (1) is $\psi$-uniformly exponentially stable.

\section{Applications}

Now we will give some examples to illustrate the theoretical result obtained.

Example 2 Consider again the delay differential equation (3). We will prove theoretically that the zero solution of (3) is $\psi$-uniformly exponentially stable, where $\psi(t)=2^{-1.22 t}$.

Let $V(x)=0.5 x^{2}$. It is easy to check the validity of condition (i) of Theorem 3 for $p=1$, $a(t) \equiv 1, b(t) \equiv 1$.

Now let $t \in \mathbb{R}_{+}$and $\phi \in C([-1,0], \mathbb{R})$ be such that $V(\psi(t) \phi(0))>0.8 V(\psi(t) \phi(s))$ for $s \in[-1,0)$ or $(\phi(0))^{2}>0.8\left(2^{-1.22 s} \phi(s)\right)^{2}$. Then applying the inequalities $(\phi(0))^{2}>$ $0.8\left(2^{1.22} \phi(-1)\right)^{2}$ and $a b \leq 0.5\left(a^{2}+b^{2}\right)$, we obtain the following inequality:

$$
\begin{aligned}
D_{(1)} V(\psi(t) \phi(0)) & =2^{-2.44 t}\left((-1.22 \ln (2)+0.5)(\phi(0))^{2}+0.5(\phi(-1))^{2}\right) \\
& <2^{-2.44 t}((-1.22 \ln (2)+0.5) 0.8+0.5)(\phi(-1))^{2} \leq 0 .
\end{aligned}
$$


According to Theorem 3 the zero solution of (3) is $\psi$-uniformly exponentially stable, i.e. the inequality $\left|2^{-1.22 t} x(t)\right| \leq\left(\left|2^{-1.22 t} \varphi\right|_{t_{0}}\right) e^{-\lambda\left(t-t_{0}\right)}$ holds, where $\lambda=0.22<-\ln 0.8$.

Also, $|x(t)| \leq 2^{1.22} 2^{1.22 t} e^{-0.22 t}\left(2^{-1.22 t_{0}}|\varphi|_{t_{0}} e^{0.22 t_{0}}\right)$, which proves that the rate of increase of any solution of (3) is smaller than $2^{1.22 t} e^{-0.22 t}$.

Now we will consider the particular case of (1) for $G(x)(t)=\max _{s \in[t-r, t]} x(s)$, i.e. we will consider differential equations with 'maxima' [14].

Example 3 Consider the following system of differential equations with maxima:

$$
\begin{aligned}
& x^{\prime}(t)=y^{2}(t)-x(t), \\
& y^{\prime}(t)=-y(t)\left(1+\max _{s \in[t-1, t]} x(s)\right) \quad \text { for } t \geq t_{0} .
\end{aligned}
$$

Note that it is not possible to obtain the solutions of the system (14) in an explicit form. So, we will use the results obtained above to draw a conclusion about the behavior of the solutions.

Let $V(t, x, y)=0.5\left(x^{2}+y^{2}\right)$.

Define $\psi_{1}(t)=\psi_{2}(t)=\frac{1}{t+2}, t \in \mathbb{R}_{+}$. Then for any $x, y \in \mathbb{R}$ and $t \in \mathbb{R}_{+}$the inequalities $0.5 \frac{1}{t+2}\left(\sqrt{x^{2}+y^{2}}\right)^{2} \leq 0.5\left(x^{2}+y^{2}\right) \leq 0.5 \frac{t+3}{t+2}\left(\sqrt{x^{2}+y^{2}}\right)^{2}$ are satisfied, i.e. condition (i) of Theorem 1 is satisfied for $p=2, a(t) \equiv 0.5$, and $b(t)=0.5(t+3)$. For any $t \in \mathbb{R}_{+}$and any function $\phi \in C\left([-1,0], \mathbb{R}^{2}\right), \phi=\left(\phi_{1}, \phi_{2}\right)$ such that $\left(\phi_{1}(0)\right)^{2}+\left(\phi_{2}(0)\right)^{2}>e^{-2}\left(\phi_{2}(s)\right)^{2}$ for $s \in[-1,0)$ we use the inequality $\max _{s \in[-1,0]} \phi_{1}(s) \geq \phi_{1}(0)$ and obtain

$$
\begin{aligned}
& D_{(14)} V(t, \phi(0)) \\
& \quad=\phi_{1}(0)\left(\phi_{2}(0)\right)^{2}-\left(\phi_{1}(0)\right)^{2}-\left(\phi_{2}(0)\right)^{2}-\left(\phi_{2}(0)\right)^{2} \max _{s \in[-1,0]} \phi_{1}(s) \\
& \quad \leq-2 V(t, \phi(0)) .
\end{aligned}
$$

According to Theorem 1 the zero solution of (14) is $\psi$-exponentially stable. From inequality (10) we obtain

$$
\frac{1}{t+2} \sqrt{(x(t))^{2}+(y(t))^{2}} \leq \sqrt{t_{0}+3}\left(\max _{s \in[-1,0]}\left\{\frac{1}{t+s+2} \sqrt{\left(\varphi_{1}(s)\right)^{2}+\left(\varphi_{2}(s)\right)^{2}}\right\}\right) e^{-\left(t-t_{0}\right)} .
$$

Therefore,

$$
\sqrt{(x(t))^{2}+(y(t))^{2}} \leq \sqrt{t_{0}+3}\left(\max _{s \in[-1,0]}\left\{\sqrt{\left(\varphi_{1}(s)\right)^{2}+\left(\varphi_{2}(s)\right)^{2}}\right\}\right) e^{-\left(t-t_{0}\right)}
$$

or

$$
\|u(t)\| \leq \sqrt{t_{0}+3}\|\psi\|_{0} e^{-\left(t-t_{0}\right)},
$$

where $u=(x, y)$.

Inequality (15) proves that the zero solution of (14) is also exponentially stable.

Now we will consider the particular case of $G(x)(t)=x(t-1)$, i.e. a nonlinear differential equation with a constant delay. 


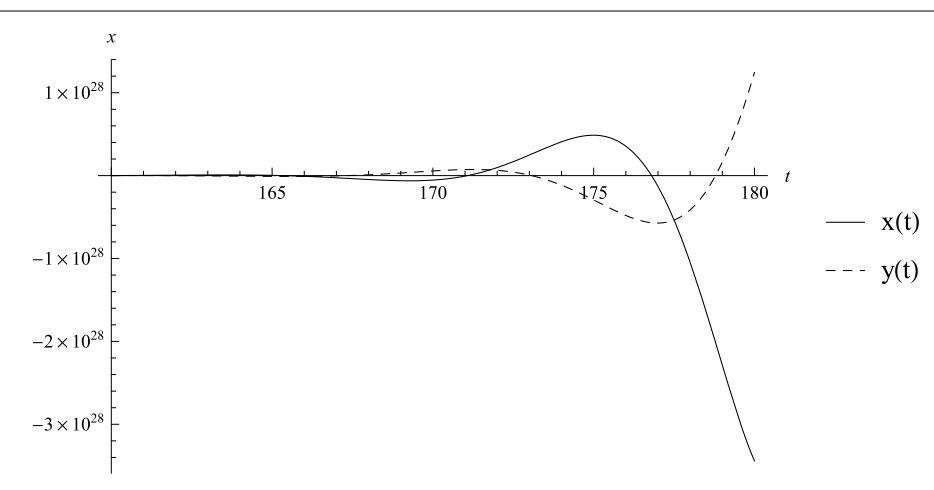

Figure 5 The graph of the solution $x(t), y(t)$ of (16), (17).

Example 4 Consider the following system of delay differential equations:

$$
\begin{aligned}
& x^{\prime}(t)=y(t)+x(t-1)+y(t-1), \\
& y^{\prime}(t)=-x(t)+x(t-1)+y(t-1) \quad \text { for } t \geq 4
\end{aligned}
$$

with initial condition

$$
x\left(t_{0}+s\right)=\phi_{1}(s), \quad y\left(t_{0}+s\right)=\phi_{2}(s) \quad \text { for } s \in[-1,0],
$$

where $x, y \in \mathbb{R}$.

We use Wolfram Mathematica to obtain numerically the solution $x(t), y(t)$ of IVP (16), (17) for $t_{0}=0, \phi_{1}(t)=1, \phi_{2}(t)=1, t \in[-1,0]$ and we graph it (Figure 5). It is obvious that the zero solution is not exponentially stable.

Applying the above results we will prove the $\psi$-exponential stability of the zero solution of (16), where $\psi(t)=\left(\psi_{1}(t), \psi_{2}(t)\right), \psi_{1}(t)=\psi_{2}(t)=e^{-6 t}$.

Consider $V(t, x, y)=0.5\left(x^{2}+y^{2}\right)$. Then the condition (i) of Theorem 3 is satisfied for $p=2, a(t) \equiv 1$ and $b(t)=1$.

For any $t \in \mathbb{R}_{+}$and any function $\phi \in C\left([-1,0], \mathbb{R}^{2}\right), \phi=\left(\phi_{1}, \phi_{2}\right)$ such that $\left(\phi_{1}(0)\right)^{2}+$ $\left(\phi_{2}(0)\right)^{2}>0.5\left(\left(\phi_{1}(s)\right)^{2}+\left(\phi_{2}(s)\right)^{2}\right)$ for $s \in[-1,0)$, we use the inequality $2 a b \leq a^{2}+b^{2}$ and obtain

$$
\begin{aligned}
D_{(16)} & V(t, \psi(t) \phi(0)) \\
= & e^{-6 t}\left(\left(\phi_{1}(0)\right)\left(\phi_{2}(0)\right)+\phi_{1}(0) \phi_{1}(-1)+\phi_{1}(0) \phi_{2}(-1)\right. \\
& \left.-\left(\phi_{1}(0)\right)\left(\phi_{2}(0)\right)+\phi_{2}(0) \phi_{1}(-1)+\phi_{2}(0) \phi_{2}(-1)\right) \\
& -3 e^{-6 t}\left(\left(\phi_{1}(0)\right)^{2}+\left(\phi_{2}(0)\right)^{2}\right) \\
\leq & e^{-6 t}\left(-2\left(\phi_{1}(0)^{2}+\phi_{2}(0)^{2}\right)+\phi_{1}(-1)^{2}+\phi_{2}(-1)^{2}\right)<0 .
\end{aligned}
$$

According to Theorem 3 the zero solution of (16) is $\psi$-uniformly exponentially stable and inequality (13) is satisfied, i.e.

$$
\|\psi(t) u(t)\| \leq\left(\|\psi \varphi\|_{t_{0}}\right) e^{-0.25\left(t-t_{0}\right)} \quad \text { for } t \geq t_{0},
$$

where $u=(x, y), x(t), y(t)$ is the solution of $(16), \lambda=0.5$. 


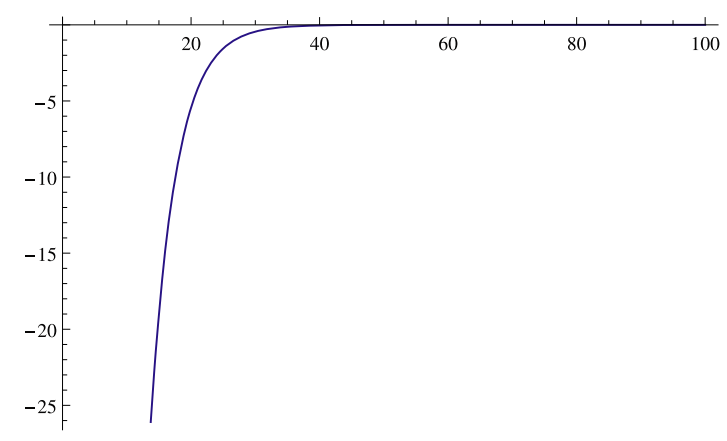

Figure 6 The graph of $e^{-6 t}(|x(t)|+|y(t)|)-2 e^{6} e^{-0.25 t}$ on $[60,100]$.

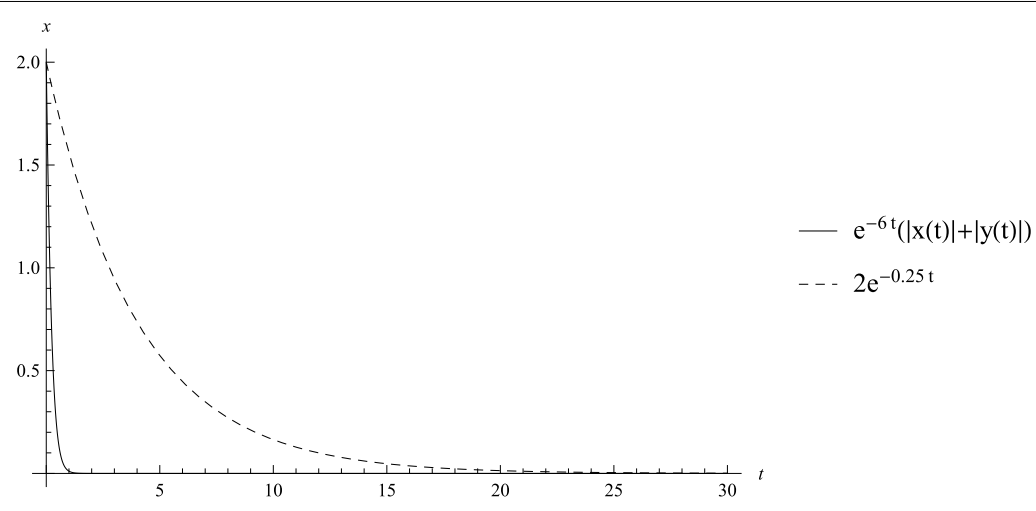

Figure 7 The graphs of $e^{-6 t}(|x(t)|+|y(t)|)$ and $2 e^{-0.25 t}$ on $[0,30]$.

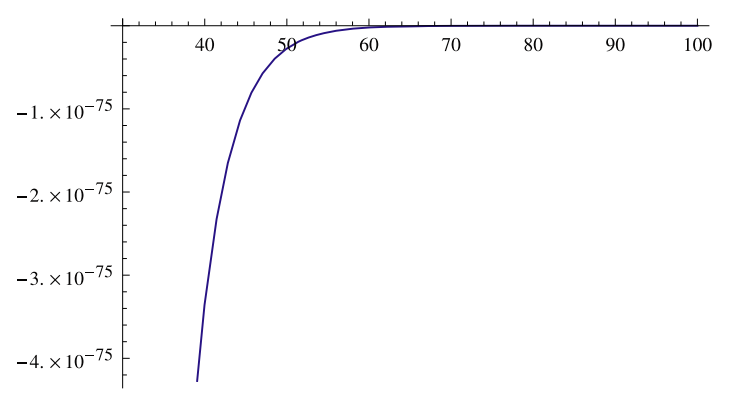

Figure 8 The graph of $e^{-6 t}(|\tilde{x}(t)|+|\tilde{y}(t)|)-150 e^{-174} e^{-0.25(t-30)}$ on $[30,100]$.

From inequality (19) it follows that $\|u(t)\| \leq e^{-5.75 t_{0}+6}\|\varphi\|_{t_{0}} e^{5.75 t}$, i.e. the rate of increase of any solution of (16) is smaller than $e^{5.75 t}$.

In the particular case of the initial condition $t_{0}=0, \phi_{1}(t)=1, \phi_{2}(t)=1, t \in[-1,0]$, inequality (19) is reduced to $\|\psi(t) U(t)\| \leq 2 e^{6} e^{-0.25 t}$, and $U=(x, y), x(t), y(t)$ is the solution of (16), (17). From Figure 6 it is seen that inequality (19) is true.

Now use the function $\psi(t)=\left(\psi_{1}(t), \psi_{2}(t)\right), \psi_{1}(t)=\psi_{2}(t)=e^{-6 t}$ as a weight and graph both $e^{-6 t}(|x(t)|+|y(t)|)$ and $2 e^{-0.25 t}$ (Figure 7). It is obvious that $e^{-6 t}(|x(t)|+|y(t)|)<e^{-0.25 t}$. 
Consider the delay equation (16) with initial condition

$$
x(t)=100, \quad y(t)=-50 \quad \text { for } t \in[29,30] .
$$

For the initial value problem (16), (20) inequality (19) is reduced to $\|\psi(t) \tilde{u}(t)\| \leq$ $150 e^{-174} e^{-0.25(t-30)}, \tilde{u}=(\tilde{x}, \tilde{y})$, where the couple of functions $(\tilde{x}(t), \tilde{y}(t))$ is the solution of (16), (20). From Figure 8 it is seen that inequality (19) is true.

\section{Competing interests}

The authors declare that they have no competing interests.

\section{Authors' contributions}

All the authors contributed equally to this work. They all read and approved the final version of the manuscript.

\section{Author details}

${ }^{1}$ Plovdiv University, Plovdiv, Bulgaria. ${ }^{2}$ Technical University of Sofia, Branch Plovdiv, Plovdiv, Bulgaria.

\section{Acknowledgements}

The research was partially supported by Project BG051P0001/3.3-05- 001 Science and Business, financed by the Operative Program, Development of Human Resources, European Social Fund and Fund Scientific Research MU13FMI002, Plovdiv University.

Received: 10 May 2014 Accepted: 4 June 2014 Published: 22 Jul 2014

\section{References}

1. Wang, Q, Zhu, Q: Razumikhin-type stability criteria for differential equations with delayed impulses. Electron. J. Qual. Theory Differ. Equ. 2013, 14 (2013)

2. Zhang, GL, Song, MH, Liu, MZ: Exponential stability of impulsive delay differential equations. Abstr. Appl. Anal. 2013, Article ID 938027 (2013). doi:10.1155/2013/938027

3. Akinyele, O: On partial stability and boundedness of degree $k$. Atti Accad. Naz. Lincei, Rend. Cl. Sci. Fis. Mat. Nat. (8) 65(6), 259-264 (1978)

4. Morchalo, J: On $\left(\psi-L_{p}\right)$-stability of nonlinear systems of differential equations. An. Ştiinţ. Univ. 'Al.ı. Cuza' laşi, Mat. 36(4), 353-360 (1990)

5. Dimandescu, A: On the $\psi$-stability of nonlinear Volterra integro-differential systems. Electron. J. Differ. Equ. 2005,56 (2005)

6. Gupta, B, Srivastava, SK: $\psi$-Exponential stability for non-linear impulsive differential equations. Int. J. Comput. Math. Sci. 4(7), 329-333 (2010)

7. Agarwal, RP, Hristova, S: Strict stability in terms of two measures for impulsive differential equations with 'supremum'. Appl. Anal. 91(7), 1379-1392 (2012)

8. Dishliev, A, Hristova, S: Stability on a cone in terms of two measures for differential equations with 'maxima'. Ann. Funct. Anal. 1(1), 133-143 (2010)

9. Henderson, J, Hristova, S: Eventual practical stability and cone valued Lyapunov functions for differential equations with 'maxima'. Commun. Appl. Anal. 14(4), 515-524 (2010)

10. Hristova, S: Lipschitz stability for impulsive differential equations with 'supremum'. Int. Electron. J. Pure Appl. Math. 1(4), 345-358 (2010)

11. Hristova, S: Practical stability and cone valued Lyapunov functions for differential equations with 'maxima'. Int. J. Pure Appl. Math. 57(3), 313-324 (2009)

12. Hristova, S: Stability on a cone in terms of two measures for impulsive differential equations with 'supremum'. Appl. Math. Lett. 23, 508-511 (2010)

13. Hristova, S, Gluhcheva, S: Lipschitz stability in terms of two measures for differential equations with 'maxima'. Int. Electron. J. Pure Appl. Math. 2(2), 1-12 (2010)

14. Bainov, D, Hristova, S: Differential Equations with 'Maxima'. CRC Press, Boca Raton (2011)

10.1186/1687-1847-2014-185

Cite this article as: Hristova and Proytcheva: Weighted exponential stability for generalized delay functional differential equations with bounded delays. Advances in Difference Equations 2014, 2014:185 